\title{
IL BELLISSIMO RI-ESSERE DI GESUALDO BUFALINO: IL NAUFRAGIO DELLA MEMORIA E LA SALVEZZA DELLA SCRITTURA (OPERE 1981-1988)
}

\author{
Alessandra Verde \\ Universidad de Sevilla
}

RESUMEN

Gesualdo Bufalino manifiesta claramente la función y del fin de sus obras y de su escritura: salvarse a si mismo del suicidio, gracias al incesante ejercicio del "ser" y del "volver a ser" que son un elemento sustancial de su narrativa. Esto lleva a una especie de auto-terapia, que recurre a la memoria como si de un territorio por explorar y analizar se tratara. El proceso conduce a la emersión del "sí mismo", del "ser" y del "baber sido" que brotan de la escritura: eso es, la cognición profunda de ficción, estilo, lenguaje y autenticidad que se enfrentan al sujeto y a su sentido de la identidad.

Palabras clave: El "sí mismo" en la escritura, Memoria, Estilística, Psicoanálisis, Metaficción.

ABSTRACT

The author plainly speaks about the function and the aims of his works and of writing: to save oneself from suicide thanks to a constant exercise of "being" and "being again" (essere e riessere) that imbues his narrative. It aims to a sort of auto-therapy that uses memory as a land to be explored and analysed. The process leads to the emerging "self" of "being" and "having been" by writing: that is, the consciousness of fiction, style, language and authenticity facing one's own identity.

Keywords: "Self" in writing, Memory, Stylistics, Psychoanalysis, Meta-fiction.

\section{LA TRAVERSATA DEL TEMPO}

"Essere o riessere, ecco il problema. La scrittura me lo risolve, mi permette di cibarmi dei miei ieri come le iene si cibano dei cadaveri e così sopravvivere nel deserto" 1 .

${ }^{1}$ Conversazione con Gesualdo Bufalino, Omicron, Roma 1996. Abbreviato a partire da qui come CGB. A parte un cenno a Calende greche (1990), le opere prese qui in considerazione e citate sono contenute nella raccolta Gesualdo Bufalino. Opere 1981-1988, (introduzione di Maria Corti, a cura di Maria Corti e Francesca Caputo), Classici Bompiani, Milano 1992. Saranno altresì abbreviati in nota i seguenti titoli: La diceria dell' untore (1981) $=D U$ e Argo il cieco(1984) $=A C$. 
Questo afferma G. Bufalino in risposta alla domanda sul perché e per chi si scrive. È un paragone piuttosto macabro ma efficace e si riferisce alla creazione letteraria o- per attenersi alla definizione dell'autore stesso-, alla creazione delle sue "fantamemorie", termine con il quale invita a guardare all'insieme delle sue opere.

Tutto ciò che stato e tutto ciò che risiede nella memoria, quella somma, e ad un tempo agglomerato fatto di "ieri", non emerge come nebbia sottile, bensì costituisce esplicitamente qualcosa -forse, un'identità-, che è in attesa di una risurrezione continua. Essa sarà crudele e dolorosa perché le è negata qualsiasi illusoria compensazione a posteriori; e malgrado ciò, il racconto del processo di ricomparsa è di per sè la misericordia del "riessere", del "miracolo del Bis, il bellissimo Riessere". La scrittura, per Bufalino, è un'autoterapia fatta per allontanare la morte, per scongiurarla, per sconfiggere il silenzio e l'amnesia. Tuttavia, ciò non significa scrivere per dimenticare e, meno ancora, riuscire a farlo. Il territorio in cui si è obbligati a muoversi è il deserto, e non si trasforma in luogo idillico in virtù dell'incantesimo della scrittura. L'esperienza che separa gli eventi (pseudo-biografici o fittizi) dalla resurrezione memoriale connota quest'ultima in termini di coscienza di una condanna umana di cui si prende atto con estrema lucidità. Si tratta, insomma, di un lasso di tempo nel quale, insieme alla memoria, è maturato il riconoscimento e lo sviluppo della riflessione che ha per oggetto quel "se stesso" che soggiace agli accidenti diegetici e narrativi: è il "se stesso" che si mette in discussione per mezzo della scrittura.

Il 'caso Bufalino', autore che conservava il suo romanzo in un cassetto e che si decise a pubblicarlo solo dopo molto tempo e dopo la tenace insistenza di Elvira Sellerio, è una circostanza che potrebbe prestarsi ad essere utilizzata per attribuire a Bufalino la natura di autore di tempo lento, dilatato; ma le ragioni della dimora paiono essere essenzialmente altre: non si è trattato di un lento cammino che ha condotto alla pubblicazione, bensì del cambiamento di opinione da parte dell'autore riguardo all'accettazione che la sua opera venisse diffusa. D'altro canto i 'casi editoriali' (e su quelli letterarî è istintiva una sincera cautela), son assai spesso casi "di società", giacché pare che il mondo tenda oggi a compiacersi di ben poche meraviglie ma di stupori d'ogni tipo; e resta il fatto che cosa ben diversa sono $i$ testi e le etichette divulgative che talvolta sembrano voler prenderne il posto.

${ }^{2}$ Ibidem 
La curiosa circostanza della comparsa tardiva dell'opera di questo autore in libreria è però evidente, e non può essere trascurata nel caso di Bufalino, per il quale il tempo è un elemento assai significativo, giacché costituisce il condizionante della memoria, la coscienza della quale promuove l'atto di scrittura. Bufalino scriveva, ma non pubblicava, a causa di un rifiuto che, egli stesso ebbe a dichiarare, si alimentava della stessa convinzione manifestata nella sua opera: "la coscienza della precarietà di ogni successo" ${ }^{3}$; ciò lo portò alla scelta di "scrivere per nessuno" ${ }^{4}$, albergando, come egli scrisse, superbia e codardia "in parti uguali" $"$ Dunque, queste sono ragioni dell'autore, circostanze appartenenti a lui solo e non questioni culturali né di mercato. È intima (e davvero non recente nella nostra tradizione letteraria più illustre, se si menziona, ad esempio Petrarca), l'attitudine a scrivere - come "ingegnosa pecchia" umanista-, per pochi e scelti lettori, considerati come eletti ed iniziati probi viri; parimenti, non esula da questa tradizione la concezione dell'opera come invenzione perpetua che solo la morte obbliga a lasciare ${ }^{6}$.

Il pudore dinnanzi al frastuono del mondo riflette l'atteggiamento, sempre vigile, del punto di vista del narratore creato da Bufalino: sfiducia continua nei confronti del reale, immersione e viaggio minuzioso nelle profondità delle debolezze umane, specialmente se si tratta delle proprie.

Tutto ciò si sviluppa nella distanza dovuta al tempo, nella forza dell'esperienza acquisita dall' io maturo e adulto, ovvero in un 'dopo' nel quale ciò che si è vissuto reclama e riceve quella specie di salvezza che concede il ritorno, una volta fissato, fermato e formato nella scrittura. Malgrado ciò, resta immutata l'assenza di misericordia, poiché non è concessa -neppure nell'illusione dell'apparenza-, la quiete dell'età matura che guarda indietro con empatica serenità.

\section{IL NAUFRAGO CRUDELE}

E proprio in tale prospettiva è trattata, di fatto, la funzione del protagonista-narratore di La diceria dell'untore (1981), il quale è destinato a portare la testimonianza del proprio passato di malato di tubercolosi. Il fatto

\footnotetext{
${ }^{3}$ CGB, p. 11.

${ }^{4}$ Ibidem

${ }^{5}$ Op.cit., p. 12.

${ }^{6}$ Ibidem
} 
stesso di essere sopravvissuto alla malattia è ciò che lo porta a considerare se stesso un traditore nei confronti di quelli che, invece, sono morti e a sentirsi in più condannato senza remissione ad essere ancora vivo. Il vivere è in fin dei conti ed inevitabilmente un vivere 'dopo', sempre segnato da un passato dal quale è impossibile affrancarsi:

Anche stanotte, cinque novembre millenovecentosettantuno, venticinquesimo anniversario della mia dimissione dalla Rocca, mi sono svegliato a metà del sonno col sapore del sangue nella gola. [...] Macchè, niente. Solo bianca, miracolosa, beneaugurante saliva. È dunque vero che sono guarito...

La storia della guarigione s'inaugura quando si ignora la possibilità di salvezza e si chiude esattamente venticinque anni più tardi. L'anniversario non rinnova la gioia di colui che ha ricevuto la grazia della guarigione, bensì ripete e richiama ancora e ancora l'incertezza che risiede nel perché delle cose: mette in discussione i concetti di giusto e ingiusto, bene e male, vero e falso ${ }^{8}$ nell'esistenza umana. Per quanto si tenti di attenersi alla realtà, la coscienza va al di là dei fatti verificabili, poiché essi sono autenticamente inscindibili da altri che li hanno preceduti e che non possono essere cancellati.

Infatti:

... la memoria s'intestardisce ancora a suscitarmi nel palato, dopo tanti anni, la minaccia di quel gusto dolcigno e fatale ${ }^{9}$.

$\mathrm{Ma}$, quale strumento possiede l'io per potersi vedere nel suo cammino vitale se non quello costituito dalla memoria e dalla rielaborazione attuale di quanto ha vissuto?

Inevitabile è il gioco al "rimpiattino fra menzogne e omissioni"10. La memoria non è sigillata, non è un regno segreto dal quale estrarre con avara parsimonia fotogrammi o vecchie immagini a proprio beneficio. Bufalino segue il percorso degli avvenimenti che si sono prodotti e li racconta da un luogo prospettico che è l' 'oggi', l' 'adesso'. Non si tratta soltanto di distanza spazio-temporale, ma di un diverso universo espressivo deliberatamente eletto. Si tratta sì, di memoria, ma di

${ }^{7} D U$, p. 134

${ }^{8}$ Vedasi anche "Benito Ciuffi, pedinatore" in L'uomo invaso (1986); qui il protagonista del racconto inizia ad essere vittima di malessere quando in lui sorge il dubbio sulla possibilità di distinguere ciò che è vero da ciò che è falso.

${ }^{9}$ Ibidem.

${ }^{10}$ Op. cit., p. 100. 
memoria scritta; vale a dire di un fenomeno che, oltre ad evocare, manifesta verbalmente.

È lecito allora chiedersi se la scrittura in Bufalino può essere presentata quale metadiscorso della memoria?

Ciò che è stato vissuto in prima persona, sommerso e poi portato al presente -coscientemente o no-, da sempre vive nella letteratura bio e autobiografica, velato in maggior o minor misura dall'istanza produttrice o dal modo in cui essa modella la materia dal punto di vista formale e stilistico.

Forse tutto o quasi tutto possiede il proprio 'meta': realtà, finzione, fisica, discorso. Forse val la pena di sottolineare in primo luogo che, nel caso che qui si considera, la memoria è previa al discorso poiché questo è portato avanti sulla memoria che è un mondo a parte, non necessariamente e mai integralmente linguistico e codificato verbalmente. $\mathrm{Ma}$ la scrittura, sì che può essere metadiscorso della memoria e, se può esserlo in Bufalino, è perché per lui la memoria mostra chiaramente di essere l'oggetto della scrittura ed il suo motore. A sua volta, il "Riessere" si manifesta come arricchimento (sebbene comporti la lucida coscienza della condanna dell'uomo) di ciò che è conosciuto e ricordato anche verbalmente. Cioè, la memoria non è mai totalmente verbale, ma lo è anche perché la verbalità può esserne forma e sostanza. Di qui che anche la scrittura possa essere un componente della memoria. Se in essa trovano posto immagini e segni, essi possono essere benissimo testi e/o lettere, parole, frasi e, insieme, unità di sensi. Un esempio: Proust compare in Bufalino non soltanto inserito in tessere di mosaico intertestuali dirette, ma in più compare per interpretare giochi evocativi che vanno al di là anche dell'interdiscorsività; ed in giochi parodici che sono qualcosa di più che semplici e brillanti boutades. Quando la narrazione riprende con ironia All'ombra delle fanciulle in fiore ${ }^{11,} \mathrm{o}$ con il medesimo tono evoca un Puck shakespeariano o l'allodola di Romeo e Giulietta $^{12}$, ciò avviene perché vi è un patrimonio memoriale nel quale le cose vissute non sono esclusivamente episodî quotidiani dell'esperienza fisica, bensì conoscenza creativa in senso ampio, competenza metabolizzata che ha alimentato il passato e ciò che appartiene all'io e al mondo sia proprio sia altrui: vita, morte, scrittura.

\footnotetext{
11 "Vedervi [...] fiorire, sfiorire, tornare a sfiorire", però le ricorda in una macchina "ad ogni impulso di tergicristallo". AC. p. 248.

12 Con un Romeo impietosamente descritto privo di qualunque attrattiva e uno straodinariamente spiccato accento trapanese. $D U$ p. 40.
} 
Quello che potrebbe essere meramente intimo e personale si eleva e abbraccia l'elemento esteriore rendendolo con-testo e sottolineandone la pluralità referenziale ed interpretativa. Si parla di un io perennemente fuori posto, solo, non spiegabile e non spiegato; si rifiuta la 'macrostoria' ${ }^{13}$ e pur tuttavia si guarda al personaggio come ad un "multiplo, un ectoplasma plurale". Tale elemento assume caratteristiche mostruose: il personaggio, dice Bufalino,

mi fuoriesce dalla bocca ed è me, non è me, è mio servo e padrone, una escrezione che, come in certi film fantascientifici dell'orrore, prima mi replica, quindi mi si rivolta e mi succhia. mi mangia, mi espelle, mi ride alle spalle e scompare ${ }^{14 .}$

Al di là della raffigurazione tra l'onirico ed il tremendista, confluiscono qui la memoria, che possiede le chiavi della ri-creazione, l'io e la modalità di rappresentazione, ovvero la scrittura letteraria che comprende l'allucinazione del quotidiano e che dipende da quanto elaborato dal processo del ricordo. Pur senza voler ridurre il tutto alla cifra di una valenza autobiografica da applicarsi obbligatoriamente e ad ogni piè sospinto nella lettura dell'autore, le circostanze invitano naturalmente ad avvicinarsi all'identità autoriale in rapporto con la realtà specifica ed originale della stesura del testo. Un nucleo narrativo o una formula o una rielaborazione espressiva che risale ad un oggetto -che si tratti di Proust o della tisi è solo congiunturale-, fa parte in modus manifesto del patrimonio memoriale dell'istanza autoriale, e l'istanza narrativa lo fonde nella costruzione del racconto con la propria forma espressiva.

Questa è una scoperta che nulla scopre; ossia: posto che contempliamo e riconosciamo l'esistenza di un contesto, posto che accettiamo socioletto ed idioletto, diamo per scontato che l'autore ricorra ancora una volta al patrimonio proprio come se fosse qualcosa di simile a ciò che lo scrittore spagnolo Juan Goytisolo paragona ad una specie di "agiografía privada”. È ovvio che contesto e formazione sono materia fondamentale per ogni autore, ma non va dimenticato

\footnotetext{
${ }^{13}$ CGB, p. 49.

${ }^{14}$ Op. cit., p. 35. L'autore sostiene che "il parziale ritorno del racconto odierno alla norma del racconto e alle sue convenzioni antiche" (e non escludiamo Bufalino, ma pensiamo, ad esempio, all'architettura de Le menzogne della notte) "non può fare a meno di deroghe e ammiccamenti palesi. [...]. La letteratura, più che vista e visibilio; sí che la mimesi più fedele non possa assolversi se non quando le sue evidenze si traducono nei modi di un'ordinaria allucinazione" (CGB passim). In tal senso ci parla la raccolta intitolata L'uomo invaso (19869, particolarmente "L'uomo invaso", " L'uscita dall'arca ovvero il Disinganno", "Le visioni di Basilio, ovvero La battaglia dei tarli e degli eroi", "Il pedinatore" e "Il ladro di ricordi".
} 
che ogni autore è davvero originale; nella sua forma espressiva, nel suo linguaggio e, insomma nel suo stile, nel suo modo di modellare la materia, secondo le proprie prescrizioni ed esigenze, per offrirla poi ad una realtà di ricettori.

La scrittura per Bufalino è capace di salvare dalla morte e la parola è capace di quasi tutto, cerca di farlo con energia inesausta. Lo scrittore non soltanto crea elaborate costruzioni espressive su un'architettura tematica. Va oltre inventando mondi di immagine e di senso grazie alla scelta di una parola che guizza dal mito alla quotidianità, fino a raggiungere il triviale. Se suggerisce un'atmosfera operistica, parla, ad esempio, per un personaggio, di una "lunga militanza di farfallone amoroso". E continua con Mozart finché gli piace; poi armonizza la figura del vecchio don Alvise di Argo il cieco e lo fa irrompere in una camera, dove due giovani conversano da soli, con l'ìmpeto "d'un basso che sorprende soprano e tenore" ${ }^{15}$. O guarda un altro personaggio con deliberato disprezzo burlesco, ritraendolo "con una cera gialla da venditore di clavatte cinese"16; e lo disorienta crudelmente tendendogli trappole che rivelano l'ignoranza (letteraria, ma non solo) dell'uomo e contemporaneamente esplicitano i referenti letterarî dell'autore: il povero direttore Biscari ascolta -senza avere la capacità di ribattere, un amico del protagonista che lamenta in un certo personaggio "una lacuna importante. La medesima di cui con Abelardo discorre con Suor Marianna Alcoforado nelle Lettere di una novizia ${ }^{117}$ : macroscopico sproposito. E ancora: la musica da ballo che proviene dalla stanza di una pensione situata su una strada statale e dove si sono rifugiati la giovane amata dal protagonista-narratore di Argo il cieco ed un ballerino, dopo essere fuggiti dal paesetto, pare un "salterio d'angelo"18 agli orecchi dell'innamorato geloso.

\section{LA SCRITTURA: SCONGIURO E SALVEZZA}

Bufalino osserva il mondo della finzione dalle profondità del proprio mondo referenziale, ma soprattutto espressivo, individuale e "multiplo". Naturalmente non viene negata la possibiltà di scegliere la prospettiva di lettura: lettura critica, interdiscorsiva, sensualista, emotiva, filologica (e per tale autore, essa può essere

\footnotetext{
${ }^{15} A C$, p. 256.

${ }^{16}$ Op.cit., p. 266. Il corsivo è nostro.

${ }^{17}$ Op.cit, p. 266.

${ }^{18}$ Op.cit, p. 259.
} 
assai stimolante); ciascuna di esse può essere riduttiva e risultare frustrante e maggiormente lo sarà nella misura in cui $\mathrm{i}$ più che possibili concorso $\mathrm{e}$ sovrapposizione di ciascuna di esse intendessero neutralizzare la profondità di prospettiva $o$, addirittura giungere a dimostrare la supremazia di un'unica visione. Il tentativo di neutralizzazione della pluridimensionalità interpretativa indurrebbe a un certo tira e molla con effetto di straniamento negativo nel lettore; questi sarebbe obbligato a costanti omissioni ricettive ed alla rinuncia al suo articolato statuto primario e legittimo di 'lettore'. È ovvio che, per ciascun caso di salto ricettivo ed interpretativo, sia esso orientato in senso filologico, critico, emotivo, etc., il lettore ha bisogno di complementi di rielaborazione e riassestamento/correzione del rapporto creatosi con il testo. D'altro canto, proprio la necessità della riflessione funge da promotrice di nuovo senso e nuovo significato nel momento in cui deve intervenire per riconoscere la metadiscorsività della scrittura sulla memoria.

La memoria non può esistere senza il tempo né sarebbe possibile descriverla o parlarne in assenza della cognizione di esso.

Barthes ricorda che il " 'tempo' è la forma timida, soffocata, della Storia, nonostante non se ne capisca il senso" ${ }^{19}$. Non si comprende il senso della Storia con l'iniziale maiuscola, tuttavia a volte noi stessi non comprendiamo nemmeno il senso della nostra storia personale, e ciò accade anche a Bufalino che manifesta la sua perplessità. La storia personale: la misuriamo, la osserviamo, la rendiamo storia perché le cose che ci sono successe ci hanno reso quelli che siamo. Abbiamo a disposizione la misura del tempo, di per sè inadeguata per spiegare il dispiegarsi dei sentimenti in un processo vitale, ma è tutto quel che abbiamo: il tempo come convenzione inevitabile che regola e giustifica -arbitrariamente- gli avvenimenti ed i cambiamenti. Al tempo si attribuisce la responsabilità di certe cose con una sicurezza che giunge ad essere una convinzione di fede. Siamo condannati al tempo, ad un'estensione che bisogna attraversare da un punto all'altro, dall'inizio alla fine (contro la quale scalciamo finché possiamo): nascita e morte.

Ciò che complica tutto è la coscienza, e tale elemento è specialmente aspro e significativo in Bufalino, che la descrive con una minuzia crudelissima nell'opera autobiografica. La coscienza è l'accettazione del passar del tempo e, insieme, riflessa nella scrittura, è quel 'ri-vivere', la resurrezione del passato come tale, però da una prospettiva diversa, matura e necessariamente impietosa. In più la

\footnotetext{
${ }^{19}$ R. Barthes, Saggi critici, Einaudi 10, 1966; Torino 1976, p. VII.
} 
coscienza rende la memoria terribile; così si sostiene ne Il malpensante: "I ricordi ci uccidono. Senza memoria saremmo immortali"20.

L'empiria dolorosa di tali postulati si manifesta in uno dei capitoli denominati "Bis", il III, di Argo il cieco, intitolato "Primo dubbio dell'autore sul libro che sta scrivendo":

Al tempo, dove sto andando? La favola mi scappa via dalle mani, la memoria mi fa la buffona dietro le spalle. Altrettanto le parole: vengono fuori storte, bistrate, beffarde; agrodolciumi volti a corrompere, come si corrompe un ragazzo, un ricordo minorenne dentro di me... Bella forza, ora che sono vecchio, farmi gioco di me da ragazzo; da furbo mago di pioggia farmi pagare le previsioni del tempo ascoltate un minuto prima alla radio. Bella forza... Ora so tutto di me, dove tendevano le linee oblique della mia sorte... ${ }^{21}$.

Interagiscono, dunque, tempo dell'esperienza e scrittura; il primo porta a "saper tutto" una volta che lo scrittore giunge alla vecchiaia, mentre la seconda, ovvero la scrittura che dovrebbe poter esprimere proprio quel "saper tutto", non ci riesce: le parole sono destinate a corrompere il ricordo. D'altro canto, la pretesa di saper tutto di se stessi non è l'esatto riflesso della realtà, ma piuttosto la patente dimostrazione di non-conformità con ciò che si presuppone che verità debba essere. La gestione della materia espressiva si fa via via più complessa e composita.

Forse, dal punto di vista della ricezione, è presente un rischio nella scrittura autobiografica ed autoreferenziale, ossia quello di una permeabilità irregolare che affonda proprio nella complessità connaturata a questa scrittura. Ma Bufalino dà un'esplicita spiegazione affermando che egli scrive per vincere la morte e non per, innanzi tutto, dilettare i suoi lettori. Inoltre, le sezioni denominate "Bis", rivolte specificamente al lettore, lo mettono in guardia contro le false aspettative che questi potrebbe alimentare:

Dunque, lettore, lasciami camminare così, spingendo avanti il mio corpo a caso, questo juke box di ricordi programmato a disubbidire. E non aspettarti da me niente che somigli a qualunque lettura ti sia mai piaciuta finora ${ }^{22}$.

La complicità del ricettore non è cercata a partire dalla empatia, bensì per opposizione. L'autore si dichiara libero da qualsiasi dovere verso chiunque si trovi al di fuori di se stesso e del suo io, rifiuta qualunque balsamo che esuli da

\footnotetext{
${ }^{20}$ Il Malpensante (1987), p. 1039.

${ }^{21} A C$, pp.262- 263. Il corsivo è nostro.

${ }^{22} A C$, p. 262.
} 
"l'arte consolatoria" della scrittura; e malgrado tutto, come si è visto, coinvolge anche il lettore. La scrittura è

un'Operetta morale con la musica di Offenbach. ${ }^{23}$

\section{Quanto scritto è:}

il dialogo di un fisico e di un metafisico arbitrato da un patafisico... un'impostura, [...], una bagatella comica che faccia velo fra me e quella tentazione antica che sai; e mi svogli l'animo dall'arcinero, dall'arcizero, dall'arciniente ${ }^{24}$.

Nella sostanza vi è una fuga dalla morte, dalla ripetuta tentazione del suicidio. Ed un invito al gioco dell' "arte arto", esclusione ed inclusione manifesta del lettore, di colui che non pare essere considerato destinatario; ecco, allora, che si esclude il lettore ed allo stesso tempo lo si coinvolge forzosamente (se no, come potrebbe mai essere in grado di identificare e riconoscere quella "tentazione antica"?). L'esperta captatio benevolentiae si trasforma in altero cenno di monito a tutto quello che avrebbe potuto costituire l'orizzonte d'attesa del lettore; infatti lo avvertiva: 'e non aspettarti da me niente che somigli a qualunque lettura ti sia mai piaciuta fino ad oggi'. E dice ancora:

L'arte arto, che ne pensi? Un arto artificiale, s'intende, e non solo per rendere più ghiotto lo scioglilingua, ma perchè questo a me veramente serve ${ }^{25}$.

Di conseguenza, è rivendicata la scrittura in funzione di colui che scrive e non certo degli altri.

Si definisce l'espressione come un 'ghiotto scioglilingua', ma la materia di cui è fatta, la parola al servizio della sostanza, si fonde in unicum di corpus tematico ed efficacia argomentativa; l'artifex domina la tecnica, ironizza con cinismo sul suo dominio stesso, cioè la scrittura, amandola in quanto

amalgama di senso e suono dentro la tazza di poche sillabe d'oro. Gremire me ne sento come una vela di vento, una voliera di voli. Ne ronzo, ne stormisco, ne sibilo... ${ }^{26}$.

e considerandola

${ }^{23}$ Jacques Offenbach (Colonia 1819-Parigi 1880), con la satira sulla Francia del II Impero, corrosivo e cinico, che si burla degli dei e della mitologia in La belle Elène (1864) serve a Bufalino come immagine del contrasto tra elementi difficilmente conciliabili, però coesistenti.

${ }^{24} A C$, p. 263. Il corsivo è nostro.

${ }^{25}$ Ibidem

${ }^{26}$ V. Calende greche (1990). 
un surrogato di vita durante il giorno e un surrogato di sonno quando non posso prender sonno, la sera ${ }^{27}$.

L' "autoterapia", ovvero la scrittura è un gesto "per metà ludico, per metà esorcistico"28 ed è un "un giocattolo" che allontana il pensiero della morte. Oramai non sembra più che si tratti di un "surrogato", di un sostituto della vita o del sonno, e sembra che si avvicini piuttosto ad un artificio in senso proprio; esso rende l'esitenza sopportabile e talvolta piacevole, perché scrivere causa piacere a Bufalino come a Leopardi del quale l'autore siciliano ricorda le note righe sulla "felicità da me provata"29.

E malgrado la creazione offra momenti di piacere, essa non cambia in modo radicale la visione del mondo; consola, conforta, ma non è in grado di rettificare nulla.

Può allora sorprendere la mancanza di fiducia nell'esistenza di ragioni plausibili che sostengano gli eventi? Non ci sono forse inganni che il tempo rivela alla collettività ed al singolo? Se la realtà è questa, non esiste possibilità di rifugiarsi in nessun luogo, di avvolgersi nella sicurezza di nessuna fede e nemmeno di nessuna ideologia. L'unica consolazione è scrivere; Bufalino scrive, come fa Leopardi, per 'essere felice', ma soprattutto per ritardare l'esecuzione, per corrompere il boia ${ }^{30}$ senza però abbandonare il "trastullo e ironia di me" 1 , dunque di se stesso.

Il problema dell'Essere o del Ri-essere si risolve nell'abbadono alla scrittura, nell'immersione in essa che consente all'autore di alimentarsi del 'ieri' e "serve

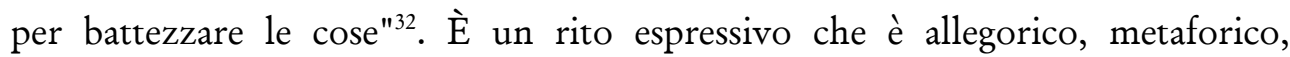
parodico e trapunto di giochi di climax ed anticlimax; è attività retorica, riconosce Bufalino, compiuta dall'artefice al quale si è fatto riferimento in precedenza, conoscitore e maestro dell'ars per mezzo della quale crea.

${ }^{27} A C$, p. 263.

${ }^{28} C G B$, p. 8.

29 "Felicità da me provata nel tempo del comporre, il miglior tempo che io abbia passato in vita mia e nel quale mi contenterei di durare finché vivo. Passar le giornate senza accorgermene e parermi le ore cortissime e meravigliarmi sovente io medesimo di tanta felicità di passione."

\footnotetext{
${ }^{30}$ CGB, p. 8

${ }^{31}$ Op.cit. , p.9.

32 ib., p. 10.
} 
L'opera che nasce non è un'astrazione isolata e remota e comprende l'invito al lettore affinché accetti un patto abilmente e -per espressa volontà virtuosistica-, rudemente presentato;

$$
\text { Continuo, allora? Continuo }{ }^{33} \text {, }
$$

propone l'autore, dopo aver sfidato i lettori dichiarandosi estraneo e in certa misura incomprensibile a se medesimo.

Non c'è altra strada se non lottare con i ricordi, con le loro bugie, le deviazioni, lottare, sanguinare e perdere. E continuare a lottare, sostiene l'autore. Ad accettare condizioni per una miseram pacem con la memoria o l'infinito della parola, Bufalino non si piega.

\section{REFERENCIAS BIBLIOGRÁFICAS}

ANONIMO, Lettere di una monaca portoghese, (a cura di Brunella Schisa), Venezia, Marsilio, 1991; (1669).

BUFALINO, Gesualdo, Opere 1981-1988, (a cura di Maria Corti e Francesca Caputo), Milano, Bompiani, 1992.

BUFALINO, Gesualdo, Calende greche: frammenti di una vita immaginaria, Milano, Bompiani 1992; (1990).

GAGLIANONE, P./TAS, L. (a cura di), Conversazione con Gesualdo Bufalino: essere o riessere, Roma, Omicron, 1996.

BARTHES, R., Saggi critici, Einaudi 1º, Torino 1976; (1963).

YATES, F. A. L'arte della della memoria, Einaudi, Torino 1985; (1966).

Alessandra Verde

averde@us.es

Depto. Filologías Integradas

Facultad de Filología

Universidad de Sevilla

C/ Palos de la Frontera s/n

41004 Sevilla (España)
Fecha de recepción: 12/09/2005

Fecha de aceptación: 11/06/2006

${ }^{33} A C$, p. 263. 